\title{
Distance doubling visual acuity test: A reliable test for nonorganic visual loss
}

\author{
Sebastian Martin Zinkernagel • Daniel Stephane Mojon
}

Received: 11 June 2008 /Revised: 23 October 2008 /Accepted: 24 November 2008 / Published online: 17 December 2008

(C) Springer-Verlag 2008

\begin{abstract}
Background To determine the value of the distance doubling visual acuity test in the diagnosis of nonorganic visual loss in a comparative observational case series.

Methods Twenty-one consecutive patients with nonorganic visual acuity loss and 21 subjects with organic visual loss as controls were included. Best corrected visual acuity was tested at the normal distance of 5 meters using Landolt Cs. The patient was then repositioned and best corrected visual acuity was tested with the previous optotypes at double the distance via a mirror.

Results Nonorganic visual acuity loss was identified in 21 of 21 patients. Sensitivity and specificity of distancedoubling visual acuity test in functional visual loss were found to be $100 \%(\mathrm{CI} ; 83 \%-100 \%)$ and $100 \%$ (CI; $82 \%-$ $100 \%$ ), respectively.

Conclusion Distance doubling visual acuity test is widely used to detect nonorganic visual loss. Our results show that this test has a high specificity and sensitivity to detect nonorganic visual impairment.
\end{abstract}

Keywords Nonorganic visual loss · Distance-doubling visual acuity test - Malingering · Psychogenic visual loss . Vision chart · Diagnostic techniques · Visual acuity

S. M. Zinkernagel $\cdot$ D. S. Mojon $(\bowtie)$

Department of Neuro-Ophthalmology and Strabismology, Kantonsspital,

St. Gallen, Switzerland

e-mail: daniel.mojon@kssg.ch

D. S. Mojon

University of Bern,

Bern CH-3012, Switzerland

\section{Introduction}

Identification of spurious ophthalmologic complaints is one of the more difficult diagnostic challenges for the ophthalmologist. One of the most common nonorganic disturbances in ophthalmology is decreased vision [1,2], which has been reported to be found in up to $7 \%$ of adults with visual complaints in an outpatient ophthalmology clinic setting [3].

Whereas nonorganic blindness may be easily uncovered by testing pupillary reactions or by evoking optokinetic nystagmus, nonorganic decreased vision is very challenging to reveal. Unilateral nonorganic visual loss is best detected by well-known tests requiring good binocular vision or tests based on blurring or occluding the better eye without the patient's knowledge [4-6]. As in unilateral blindness, the absence of a relative afferent pupillary defect may indicate nonorganic decreased vision. However, if the patient displays symmetrically decreased vision, there are only a few objective tests to reveal a nonorganic cause, and it may require considerable time and inventiveness to diagnose this condition. Yet this form of visual loss requires the greatest sacrifice of everyday life function on the part of the patient. These patients often display a discrepancy between the manner of ambulation and visual acuity (VA), and thus arouse suspicion of faking their symptoms. For such cases a test first described by Helmbold in 1896 [7] is widely used. The test, which is also useful for unilateral nonorganic visual loss, makes use of the fact that if the distance to the reading chart is doubled with help of a mirror, the given stimulus will appear half as large, since the visual angle is cut in half. Hence the patient will only be able to read letters twice as large as the original (for example the 20/80 line instead of the 20/40 line) at double the original 
distance. However if the patient is able to read the same line at double the distance, his actual VA is much better (for example if he is able to read up to $20 / 40$ at the normal distance and still recognizes this line at double distance, his VA is 20/20).

We have named the test "distance-doubling visual acuity test", because "mirror test" usually denotes the test for detection of nonorganic blindness, where eye movements are triggered when a mirror is moved in front of the malingering patient. When performing this test, care has to be taken to avoid learning effects, since patients with organic visual loss would otherwise be able to read the same line at double the distance. Although the test has been in use for over a century, the effectiveness of this test has, to our knowledge, never been investigated so far. In this study we assessed the reliability of this test in a clinical setting.

\section{Material and methods}

The research protocol followed the Tenets of the Declaration of Helsinki. Ethics Committee approval was not required for this study. All patients had a full neuro-ophthalmologic work-up.

Forty-seven patients with decreased VA (range 0.050.7) referred to the Neuro-Ophthalmologic Service at the Kantonsspital St. Gallen, Switzerland were evaluated. Inclusion criteria: all patients seen by the author DSM in the years 2005 to 2007 having presumed nonorganic VA loss were included. In the same time period, patients with decreased organic VA were included as controls. Patients with abnormal eye movements, refractive errors of greater than 5 spherical equivalents or astigmatism exceeding 2.5 diopters, reduced general health conditions or having difficulties in communicating with the health personnel were excluded. Five patients with nonorganic loss of visual acuity had to be excluded because the patients were lost for follow up.

Twenty-one patients (mean age: 22.8 years, range $7-$ 52 years) had nonorganic VA reduction (mean VA: 0.31 range: $0.05-0.7)$. Of these 21 patients, five presented with monocular visual loss and 16 with symmetrical decreased vision. Twenty-one patients (mean age: 30 years, range 4 68 years) had diminished organic VA (mean VA: 0.31, range: $0.05-0.7$ ). Of these 21 patients, 20 had unilateral VA loss and one had bilateral VA loss.

The diagnosis of nonorganic visual acuity loss was assured by a complete, normal neuro-ophthalmic examination, and by better VA verified by one or several of the following tests:

By starting with very small optotypes and displaying disbelief that the patient is not able to read, and proceed until he reads (for unilateral and symmetric bilateral VA loss).

By using an optotype chart for detection of nonorganic visual loss designed by DSM [8] (for unilateral and symmetric bilateral VA loss).

By using a polarizing test, with each eye seeing different portions of the eye chart. Polarized numbers at a distance of $5 \mathrm{~m}$ were presented (Topcon Auto Chart Projector ACP-6, Topcon Inc., Paramus, NJ, USA) and patients were asked to read all visible optotypes.

To exclude visual field defects, kinetic Goldmann perimetry was performed in all patients older than 10 years of age. Careful confrontative visual field examination was accomplished in younger patients.

The following examination procedure, which was always performed by the same investigator (DSM), was used: VA was tested on each eye separately by presenting standardized Landolt $\mathrm{Cs}$ at a distance of 5 meters (16.4 feet) and forced choice procedure (Fig. 1a). The patient was thoroughly instructed about how to read the optotypes. The subject had to try to identify or otherwise guess the direction of the $\mathrm{C}$ and the line was noted in which the patient could still read 4 of 4 presented optotypes. The patient was then asked to move to a pre-defined point at the level of the Landolt chart, from which point he was able to see the mirror. It is important that there is an angle between the chart, the mirror and the patient in order for the patient not to see himself in the mirror, so as not to arouse the patient's suspicion. Additionally the patient was then instructed in the following way about the test: "We will retest your VA with a special filter, which often helps to see more sharply". The previous clearly legible Landolt C line was now twice as far away from the patient. Thus the Cs

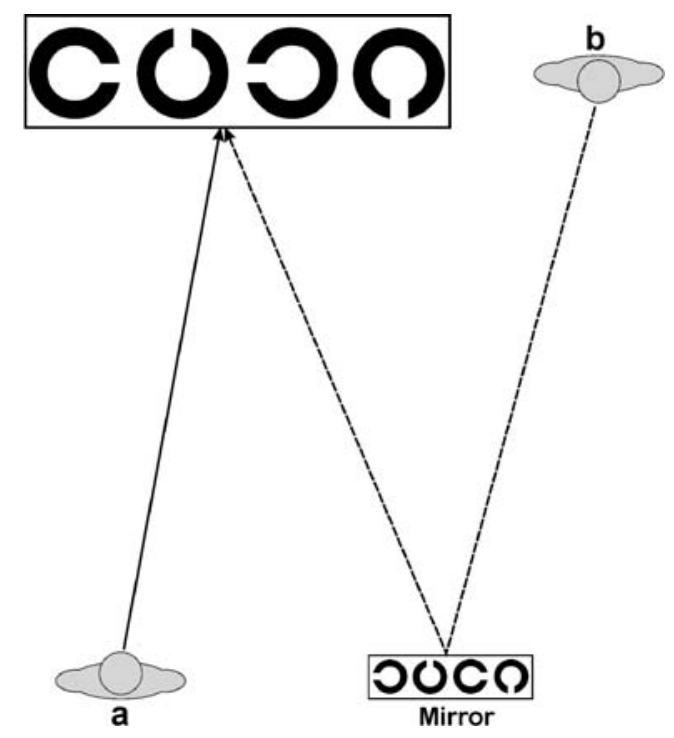

Fig. 1 VA at $5 \mathrm{~m}$ (a). Distance doubling visual acuity at $10 \mathrm{~m}$ through mirror (b) 
were half the size. The patient was then asked to read the same line via the mirror again using a forced-choice strategy (Fig. 1b). In addition the patient was asked whether the filter helped to see the optotypes more clearly. The whole examination was performed with dimmed lights in order to avoid the patient seeing a mirror image of the room, which again might arouse suspicion.

Since reading again the same optotypes at double the distance corresponds to a doubling of visual acuity, it is unlikely that learning effects will strongly affect this test. However, in order to avoid as much as possible a learning effect, the visual acuity testing procedure has to be carefully explained at the beginning. Additionally, when testing at the threshold at single distance, patients should be persuaded to try to read the optotypes claimed to be unrecognizable. For visual acuity tests, determination of repeatability is important, since optotype design, fatigue and learning effects may influence the results. Measuring of the repeatability of tests by the same as well as by other examiners is important when assessing a diagnostic test. We addressed this by testing inter- and intraobserver variation in a total of seven patients. Three of these patients had normal and four had a decrease of VA due to refractive changes. In the latter, full visual acuity could be achieved with corrective lenses, making a nonorganic component highly unlikely. Three different observers were involved to test for interobserver variation, and intraobserver variation was tested on three different days.

\section{Results}

Table 1 summarizes the visual acuity findings of patients with nonorganic visual loss. In the first column, visual acuity obtained with the forced-choice strategy is summarized. The second column displays the VA obtained via the mirror, which is twice the distance and thus double the VA. The last column summarizes follow up VA providing an additional indication for nonorganic visual acuity loss.

In the group with organic visual loss, none of the patients could read the smallest line when looking at the Landolt $\mathrm{Cs}$ at double the distance via the mirror and 21 out of 21 of patients $(100 \%)$ were unable to see this line at double the distance. In this group, $12 \%$ had bilateral visual loss and $88 \%$ had monocular visual loss. Diagnosis in the group with organic VA loss was as follows: six had refractive errors, five had proven amblyopia, four had retinopathies and six had optic nerve neuropathies.

In the group with nonorganic visual field loss, 21 out of 21 patients $(100 \%)$ were able to read all Landolt Cs at double the distance. $82 \%$ had bilateral visual loss, and $18 \%$ had monocular decreased VA. In pure nonorganic VA loss, distance-doubling VA was at least twice the claimed VA.
Table 1 Column 1: Claimed visual acuity (VA), and column 2: VA at double distance in nonorganic visual loss

\begin{tabular}{lll}
\hline 1 & 2 & 3 \\
\hline Best claimed VA & Mirror VA & $\begin{array}{l}\text { Best VA by other } \\
\text { means/ after follow up }\end{array}$ \\
0.2 & & 0.8 \\
0.2 & 0.4 & 0.8 \\
0.08 & 0.4 & 1.0 \\
0.3 & 0.16 & 1.0 \\
0.6 & 0.6 & 1.0 \\
0.4 & 1.2 & 1.0 \\
0.2 & 0.8 & 0.9 \\
0.3 & 0.4 & 0.5 \\
0.2 & 0.6 & 0.4 \\
0.1 & 0.4 & 0.3 \\
0.5 & 0.2 & 0.9 \\
0.4 & 1.0 & 1.0 \\
0.4 & 0.8 & 0.8 \\
0.4 & 0.8 & 0.9 \\
0.4 & 0.8 & 1.0 \\
0.3 & 0.8 & 0.7 \\
0.1 & 0.6 & 1.0 \\
0.2 & 0.2 & 1.0 \\
0.6 & 0.4 & 1.0 \\
0.1 & 1.2 & 0.6 \\
0.5 & 0.2 & 0.9 \\
\hline
\end{tabular}

The best VA of either eye is listed. Column 3 shows best VA after follow-up (range 1-12 months)

Five of these patients stated that the filter helped to see the optotypes at double distance more clearly, and 17 stated that visibility was equal. None of the patients found that visibility had decreased. All of the patients showed a significantly better visual acuity after 3 weeks to 12 months. The neuro-ophthalmic examination remained normal.

Sensitivity was $100 \%$ (CI; $82 \%-100 \%)$. Specificity was $100 \%$ (CI; $83 \%-100 \%$ ). Positive and negative predictive values of distance-doubling VA in functional visual loss were found to be $100 \%$.

The test proved to have a very high threshold for false positive results, since doubling the distance led to inability of a normal patient to recognize the optotypes. Intra- and interobserver repeatability of the test as well as reliability at two levels of VA also showed consistent results. However, because of the limited number of patients and observers included, some degree of intra- and interobserver variation cannot be excluded.

\section{Discussion}

Nonorganic visual loss refers to variable loss of vision in one or both eyes that cannot be explained by an adequate 
organic cause. Thus, disturbances in the ocular media, refractive errors, macular diseases or dysfunction of the optic nerve or visual pathway have to be excluded, before even considering this diagnosis. The broad array of possible causes for visual loss makes the diagnosis of nonorganic visual loss very challenging. Possible hints towards this diagnosis may be obtained by meticulously taking the patient's history and adhering to any inconsistencies. Further information can be obtained by observing the patients' movements. However, to be able to prove that the alleged visual loss is imagined, the ophthalmologist must have an objective and reproducible test.

We describe such a test based on the concept that a 1-minute angle represents normal VA. If the optotype distance is varied, the visual angle is varied as well, meaning that a certain angle is half the size at double distance. In our experience, it is important to instruct the patient that his VA will be tested with a special filter. This will motivate the patient, and may help lessen his suspicion. One should also avoid testing at the absolute threshold distance, in order for the test to be more reliable. It is very important to use Landolt Cs or Snellen Es because the mirror inverts normal letters or numbers and may give the patient a hint towards the real nature of the test. It is also important to use dimmed light, because the patient may recognize surrounding objects through the mirror, which might arouse suspicion. Helmbold used the test with numbers. In order to allow reading the numbers normally, half of the numbers were inverted.

The distance doubling VA test has some limitations and shortcomings. It is not useful in patients with severe nonorganic visual loss who claim not to see even the largest optotypes. It may also produce false positive results in younger hyperopic patients, because of their ability to accommodate. In our experience the mirror often represents a strong accommodation stimulus, and the hyperopic patient can often read the optotypes at double the distance via the mirror.
Finally, we would like to point out that our technique must be used as an extension of the complete neuro-ophthalmologic examination including, if necessary other examinations such as electrophysiological tests [9], keratography, and brain imaging.

In conclusion, using the distance-doubling VA test in patients referred because of unclear VA proved to be a very reliable diagnostic tool to discriminate between organic and nonorganic visual loss.

Funding The study was partly funded by the OPOS Foundation, Stiftung zugunsten von Wahrnehmungsbehinderten, St. Gallen, Switzerland

\section{References}

1. Kathol RG, Cox TA, Corbett JJ, Thompson HS (1983) Functional visual loss: follow-up of 42 cases. Arch Ophthalmol 101:729-735

2. Keltner JL, May WN, Johnson CA, Post RB (1985) The California syndrome: functional visual complaints with potential economic impact. Ophthalmology 92:427-435

3. Ramke J, Palagyi A, Naduvilath T, du Toit R, Brian G (2007) Prevalence and causes of blindness and low vision in TimorLeste. Br J Ophthalmol 91(9):1117-1121, doi:10.1136/bjo.2006. 106559

4. Miller BW (1973) A review of practical tests for ocular malingering and hysteria. Surv Ophthalmol 17:241-246

5. Miller NR, Keane JR (1998) Neuro-ophthalmologic manifestations of nonorganic disease. In: Miller NR, Newman NJ (eds) Walsh and Hoyt's Clinical Neuro-ophthalmology, vol. 1, 5th edn. Williams \& Wilkins, Baltimore, pp 1765-1786

6. Keane JR (1982) Neuro-ophthalmic signs and symptoms of hysteria. Neurology 32:757-762

7. Helmbold X (1896) Ueber simulation. Monatsbl Augenheilkd $34: 217-218$

8. Mojon DS, Flueckiger P (2002) A new optotype chart for detection of nonorganic visual loss. Ophthalmology 109(4):810-815, doi:10.1016/S0161-6420(01)01050-8

9. Bach M, Maurer JP, Wolf ME (2008) Visual evoked potential-based acuity assessment in normal vision, artificially degraded vision, and in patients. Br J Ophthalmol 92(3):396-403, doi:10.1136/ bjo.2007.130245 